\title{
Approximation of Conservative Fields and the Element 'Edge Shape Matrix'
}

\author{
Igor Tsukerman \\ Electrical Engineering Department, The University of Akron, OH 44325-3904, USA
}

\begin{abstract}
The accuracy of finite element approximation on tetrahedral elements is studied using the previously derived maximum eigenvalue condition. This condition is linked with the minimum singular value of the element 'edge shape matrix' that characterizes the flatness of an element. A geometric interpretation of these results is discussed.

From the theoretical viewpoint, a better insight into the mechanism of approximation errors is gained. From the practical perspective, a precise characterization of shape of tetrahedral elements becomes possible.
\end{abstract}

Index terms - finite elements, tetrahedral mesh, error estimate, approximation, interpolation, shape, singular value

\section{INTRODUCTION}

The accuracy of Finite Element (FE) approximation depends on the size and shape of elements. For example, it is well known from the engineering practice and from the angle conditions [1-3] that first order triangular elements, frequently used in 2D computation, should not be "too flat".

A new general criterion of approximation accuracy is proposed in [4]. It is based on the maximum eigenvalue of a (scaled) element stiffness matrix and is valid for arbitrary 2D and 3D elements satisfying a few general assumptions. Unlike the angle conditions for triangles, the eigenvalue criterion is algebraic. However, it is worthwhile establishing its geometric meaning in some familiar cases.

For first order triangular node elements, the maximum eigenvalue criterion leads to Zlámal's minimum angle condition [1]. For first order tetrahedral elements, this criterion yields an angle condition between edges and faces [4].

The present paper shows that for approximation of conservative fields on Whitney edge elements, the maximum eigenvalue criterion [4] yields a very precise characterization of tetrahedral shape. Since Whitney element representation of a conservative field is equivalent to the first order nodal representation of the potential, this result applies to first order nodal elements as well.

Essential for the analysis is the element 'edge shape matrix' $E$ introduced in the following section. The minimum singular value of this matrix provides the necessary link between algebraic and geometric conditions. While characterizing the geometric "flatness" of a tetrahedral element, this singular value is also directly related to the maximum eigenvalue of the scaled stiffness matrix on which the new FE accuracy criterion [4] is based.

In Section II, the element edge shape matrix is introduced.

Manuscript received November 3, 1997. The work was supported in part by the National Science Foundation research grant ECS-9702364.

I. Tsukerman, itsukerman@uakron.edu, http://www.ecgf.uakron.edu/ igor
In Section III, the maximum eigenvalue criterion is briefly reviewed. Then the connection between the singular value of the shape matrix and the maximum eigenvalue of the scaled stiffness matrix is analyzed for edge elements on tetrahedra (Section IV). Links with other geometric / algebraic parameters of a tetrahedral element are discussed in Section V. A numerical example is presented in Section VI.

\section{The Element 'EdGe Shape MATRIX'}

The shape of a tetrahedron can be characterized by six unit vectors $e_{1}, \ldots, e_{6}$ directed along the edges (Fig. 1). The $3 \times 6$ matrix $E$ whose columns are the $e_{i}$ vectors will be called the element 'edge shape matrix $E$ ', or just the $E$-matrix:

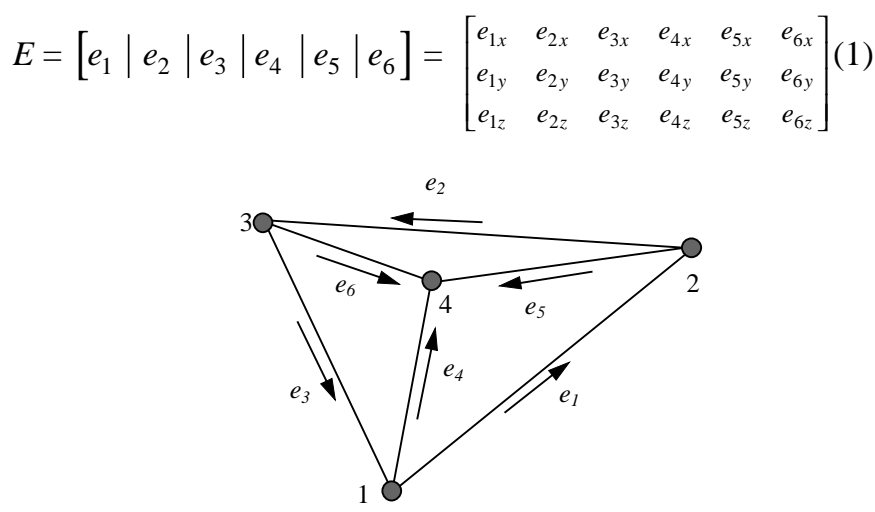

Fig. 1. Unit edge vectors $e_{i}$ characterize the shape of a tetrahedron.

Let $v \in R^{3}$ be an arbitrary vector with the Cartesian components $v_{x y z}=\left(v_{x}, v_{y}, v_{z}\right)^{T}$, and let

$v_{e}=\left(v_{e 1}, v_{e 2}, v_{e 3}, v_{e 4}, v_{e 5}, v_{e 6}\right)^{T} \equiv\left(\boldsymbol{v} \cdot \boldsymbol{e}_{1}, \boldsymbol{v} \cdot \boldsymbol{e}_{2}, \boldsymbol{v} \cdot \boldsymbol{e}_{3}, \boldsymbol{v} \cdot \boldsymbol{e}_{4}, \boldsymbol{v} \cdot \boldsymbol{e}_{5}, \boldsymbol{v} \cdot \boldsymbol{e}_{6}\right)^{T}$

be the $R^{6}$-vector of projections of $v$ on the $e$-vectors. The transformation between $v_{x y z}$ and $v_{e}$ is given by

$$
v_{e}=E^{T} v_{x y z}
$$

The shape matrix $E$ has the singular value decomposition [5]

$$
E=P \Sigma Q^{T}
$$

where $P$ is a $3 \times 3$ orthogonal matrix, $Q$ is a $6 \times 6$ orthogonal matrix, and

$$
\Sigma=\left[\begin{array}{cccccc}
\sigma_{1}(E) & 0 & 0 & 0 & 0 & 0 \\
0 & \sigma_{2}(E) & 0 & 0 & 0 & 0 \\
0 & 0 & \sigma_{3}(E) & 0 & 0 & 0
\end{array}\right]
$$

The singular values $\sigma_{1} \geq \sigma_{2} \geq \sigma_{3} \geq 0$ are equal to the square roots of the eigenvalues of matrix $E E^{T}$ [5]:

$$
\sigma_{i}^{2}(E)=\lambda_{i}\left(E E^{T}\right), \quad i=1,2,3
$$


A geometric interpretation of the minimum singular value follows from the vector component transformation (2):

$$
\begin{aligned}
\left\|v_{e}\right\|_{E^{6}}^{2} & \equiv\left(v_{e}, v_{e}\right)_{E^{6}}=\left(E^{T} v_{x y z}, E^{T} v_{x y z}\right)_{E^{6}} \\
& =\left(E E^{T} v_{x y z}, v_{x y z}\right)_{E^{3}} \geq \lambda_{\min }\left(E E^{T}\right)\left\|v_{x y z}\right\|_{E^{3}}^{2}
\end{aligned}
$$

It follows from (4), (5) that

$$
\sigma_{\min }(E) \leq\left\|v_{e}\right\|_{E^{6}} /\left\|v_{x y z}\right\|_{E^{3}} \text { for any nonzero } v_{x y z} \in R^{3},
$$

where an exact equality is achieved when $v_{x y z}$ is the minimum eigenvector ${ }^{1}$ of $E E^{\mathrm{T}}$. Thus

$$
\sigma_{\min }(E)=\min _{\left\|v_{x y z}\right\|_{E^{3}}=1}\left\|v_{e}\right\|_{E^{6}}
$$

Fig. 2 illustrates the geometric meaning of a "small" $\sigma_{\min }$. If the system of vectors $e_{1}, \ldots, e_{6}$ is close to being coplanar, there exists a unit vector $v$ that is "almost orthogonal" to all of the $e$ vectors, i.e. has very small projections on all edges, and consequently $\sigma_{\min }$ in (6) is small. A large approximation error that happens to lie in the direction of such a vector $v$ will have small edge components.

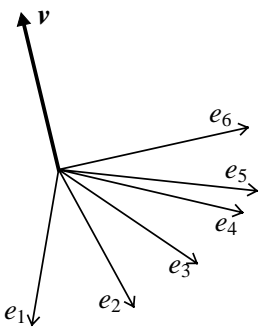

Fig. 2. A vector "almos orthogonal" to "almost coplanar" edge vectors
If any three of the $e_{i}$ vectors are linearly independent, a nonzero vector $v$ cannot be orthogonal to all three of them, and therefore $v_{e}$ must be nonzero and $\sigma_{\min }(E)$ in (6) is strictly positive. In other words, the minimum singular value $\sigma_{\min }$ is zero if and only if all six $e_{i}$ vectors are coplanar. In general, the minimum singular value characterizes the "level of degeneracy", or flatness, of a tetrahedron.

At the same time, it is shown in Section IV that $\sigma_{\text {min }}(E)$ is related to the maximum eigenvalue of the (scaled) edge element stiffness matrix; this eigenvalue, according to [4], characterizes the accuracy of FE approximation.

\section{THE MAXIMUM EIGENVALUE CRITERION}

In FEM the exact scalar or vector potential or field $u_{*}$ is approximated by an FE function $\tilde{u}=\sum_{1 \leq i \leq n} u_{i} \phi_{i} \in \tilde{W}$, where $\left\{\phi_{i}\right\}$ is a system of FE basis functions (node or edge based), and $\tilde{W}$ is the finite dimensional space spanned by these functions; $u=\left(u_{1}, u_{2}, \ldots, u_{n}\right)^{T} \in R^{n}$ is a vector of FE degrees of freedom (d.o.f.), e.g. node or edge values. The error of approximating $u_{*}$ by functions in $\tilde{W}$ is

$$
\varepsilon_{a} \equiv \min _{\widetilde{u}_{a} \in \tilde{W}}\left\|\tilde{u}_{a}-u_{*}\right\|_{E}
$$

\footnotetext{
${ }^{1}$ I.e. the eigenvector corresponding to the minimum eigenvalue.
}

where the subscript " $E$ " indicates an appropriate energy norm. In the FE space $\tilde{W}$, the energy norm is induced by the stiffness matrix $A$, i.e. $\|\tilde{u}\|_{E}^{2}=(A u, u)_{E^{n}}, \quad \tilde{u} \in \tilde{W}$.

The global stiffness matrix $A$ is the sum of the stiffness matrices $A^{(e)}$ of individual elements $(e)$. The following error estimate $^{2}$ is derived in [4]:

$$
\varepsilon_{a}^{2} \leq c \sum_{(e) \in \text { mesh }} h^{(e) 2 \kappa} \lambda_{\max }\left(A^{(e)}\right)
$$

Here the constant $c$ may depend only on $u_{*}$ but not on the maximum element size $h^{(e)}$ and shape; $\lambda_{\max }\left(A^{(e)}\right)$ is the largest eigenvalue of the stiffness matrix of an element $(e)$ :

$$
\lambda_{\max }\left(A^{(e)}\right)=\max _{\|x\|_{2}=1}\left(A^{(e)} x, x\right)
$$

Finally, $\kappa$ in (7) is the order of approximation of the FE degrees of freedom. For example, $\kappa=2$ when the d.o.f.'s are the nodal values and the basis functions are piecewise-linear.

Error estimate (7) is global, but each individual term of the sum in the right hand side represents a local (elementwise) estimate. Analysis of approximation errors is to a large extent reduced by (7) to evaluating maximum eigenvalues of element stiffness matrices. (Alternatively, matrix traces can be computed instead of the maximum eigenvalues.)

\section{EdGE ELEMENT APPROXIMATION}

Edge element basis functions $w_{k}$ are expressed via standard nodal basis functions $\phi_{i}$ as $w_{k}=\phi_{i} \nabla \phi_{j}-\phi_{j} \nabla \phi_{i} \quad$ [6].

Instead of the usual degrees of freedom, the circulations of the vector field along the edges, we consider as d.o.f.'s the tangential components of $\boldsymbol{H}$ at edge midpoints: $H_{e i} \equiv \boldsymbol{H} \cdot \boldsymbol{e}_{i}$. This variable change amounts to multiplying the basis functions by edge lengths $l_{k}: w_{k}^{\prime}=w_{k} l_{k}$. We focus on the special but important case of conservative fields: $H_{*}=\nabla u_{*}$ and $\tilde{H}=\nabla \tilde{u}$. In this case, edge elements may be viewed just as a means of studying the node element approximation. Approximation of non-conservative fields by edge elements requires separate analysis.

The approximation error is measured in the energy norm

$$
\|H\|_{E}=\left(\int_{\Omega}|H|^{2} d V\right)^{1 / 2}
$$

The entries of the element stiffness matrix corresponding to this energy norm are

$$
A_{i j}^{(e)}=\int_{(e)} w_{i}^{\prime} \cdot w_{j}^{\prime} d V
$$

A piecewise-linear approximation $\tilde{u}$ of the exact scalar potential $u_{*}$ on first order tetrahedral elements is equivalent to a piecewise-constant approximation $\tilde{H}$ of the $H$ field; i.e. $\tilde{H}^{(e)}$ is constant. Let $\tilde{W}_{\text {const }}^{(e)}$ be the space of vector fields

2 Presented here in a slightly simplified form. Only the dominant term relevant to the present analysis is retained. 
defined within a particular element $(e)$ and constant over that element. The maximum eigenvalue of the operator $A^{(e)} r e$ stricted to $\tilde{H} \in \tilde{W}_{\text {const }}^{(e)}$ is ${ }^{3}$ (note that $H_{e}=E^{T} H_{x y z}$ (2))

$$
\begin{aligned}
\lambda_{\max }\left(A^{(e)} \mid \tilde{H} \in \tilde{W}_{c o n s t}^{(e)}\right)=\max _{H_{x y z} \in E^{3}}\left(A^{(e)} H_{e}, H_{e}\right)_{E^{6}} /\left(H_{e}, H_{e}\right)_{E^{6}} \\
=\max _{H_{x y z} \in E^{3}}\left(\int_{(e)} \tilde{H}^{2} d V /\left\|H_{e}\right\|_{E^{6}}^{2}\right) \\
=\max _{H_{x y z} \in E^{3}}\left(\left\|H_{x y z}\right\|_{E^{3}}^{2} /\left\|H_{e}\right\|_{E^{6}}^{2}\right) V^{(e)}=\sigma_{\min }^{-2}(E) V^{(e)}
\end{aligned}
$$

where $V^{(e)}$ is the element volume. Therefore, since the order of approximation of $H_{e}$ 's is $\kappa=1$, the error estimate (7) can be rewritten in terms of $\sigma_{\min }(E)$ :

$$
\varepsilon_{a}^{2} \leq c \sum_{(e)} h^{(e) 2} \sigma_{\min }^{-2}\left(E^{(e)}\right) V^{(e)}
$$

This is a global error estimate, but each individual term in the sum represents a (squared) element-wise error.

\section{LINKS WITH OTHER MEASURES}

\section{A. The Face Shape Matrix}

In addition to the edge shape matrix $E$, one may consider the $3 \times 4$ 'face shape matrix' $G$ whose columns are the (outward) unit normal vectors to the four faces of the tetrahedron. The minimum singular value $\sigma_{\min }(G)$, as well as $\sigma_{\min }(E)$, characterizes the level of 'flatness' of the element. However, as shown in Appendix I, a criterion based on $\sigma_{\min }(G)$ (if such a criterion were valid) would be more restrictive than the $\sigma_{\min }(E)$-criterion.

\section{B. The Inscribed Sphere Criterion}

The singular value criterion of the flatness of a tetrahedron is less restrictive than the ratio of the radius of the inscribed sphere $r$ to the maximum element edge $l_{\max }$. In fact,

$$
\sigma_{\min }(E) \geq r / l_{\max }
$$

Proof. Let $z_{i}(i=1,2,3,4)$ be the $z$-coordinates of the tetrahedral nodes. Assume without loss of generality that the first node is placed at the origin and that the tetrahedron is scaled to $l_{\max }=1$ and rotated to have the minimum unit eigenvector $\boldsymbol{v}$ of $E E^{T}$ run along the $z$-axis. According to (6),

$$
\begin{array}{r}
\sigma_{\min }^{2}(E)=\left\|v_{e}\right\|_{E^{6}}^{2}=\sum_{1 \leq j<i \leq 4}\left(v \cdot e_{j i}\right)^{2} \geq \sum_{2 \leq i \leq 4}\left(v \cdot e_{1 i}\right)^{2}= \\
=\sum_{2 \leq i \leq 4} z_{i}^{2} / l_{1 i}^{2} \geq \sum_{2 \leq i \leq 4} z_{i}^{2}
\end{array}
$$

where each edge is now labeled by its two end nodes; $l_{1 i}$ is the length of the edge connecting nodes 1 and $i, l_{1 i} \leq l_{\max }=1$.

\footnotetext{
3 With some abuse of notation. The 'maximum eigenvalue in the subspace' is understood as the maximum value (8) in the subspace rather than the parameter in $A x=\lambda x$.
}

The first summation in the chain of (13) is over all six edges $j \rightarrow i$, while the subsequent summations are over three nodes $i=2,3,4$ and the corresponding edges $1 \rightarrow i$.

It immediately follows from (13) that for all nodes $\left|z_{i}\right| \leq \sigma_{\min }$. The scaled tetrahedron therefore lies entirely between the planes $z= \pm \sigma_{\min }$; hence $r \leq \sigma_{\min }$. $\diamond$

At the same time, the converse statement that $\sigma_{\min }(E) \leq$ $\mathrm{cr} / l_{\max }$ is not true. Consider a 'right' tetrahedron with three mutually orthogonal edges, two of these edges being of unit length and the third one tending to zero. Then the radius of the inscribed sphere tends to zero, while the minimum singular value $\sigma_{\min }(E)$ remains equal to one (see [7]).

\section{The Solid Angles}

The 'level of linear independence' of three unit edge vectors of a tetrahedron can be characterized by the trihedral (solid) angle formed by these vectors, or, alternatively, by the minimum singular value of the edge shape matrix (more precisely, by the $3 \times 3$ submatrix associated with the three edges).

This observation suggests that a connection exists between the minimum singular value and the solid angle. Indeed, the argument in Section V-B shows that for a sequence of tetrahedral elements with $\sigma_{\text {min }}(E)$ tending to zero, all faces and edges will in the limit lie in the same plane (perpendicular to the minimum eigenvector of $E E^{T}$ ). Therefore all solid (resp. dihedral) angles will tend to zero or $2 \pi$ (resp. zero or $\pi$ ). A more detailed analysis is presented in [8]. It is also evident that the minimum singular value condition is much less restrictive than the requirement [9] that all dihedral angles be acute.

\section{NUMERICAL ILLUSTRATION}

A series of numerical tests for the Laplace equation in a unit cube was conducted. Inhomogeneous Dirichlet boundary conditions were consistent with the exact solutions specified in Appendix II. Two coarse meshes were generated using the commercial FE software package Cosmos ${ }^{\mathrm{TM}}$ and were subsequently refined. One of the initial meshes is shown in Fig. 3.

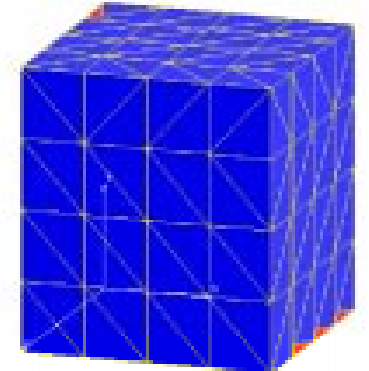

Fig. 3. A typical initial mesh for test problems.
The trace of $\left(E E^{T}\right)^{-1}$ was calculated in lieu of $\sigma_{\text {min }}^{-2}(E)$ in the global error estimate (11). In Fig. 4 this estimate is plotted vs. the actual error. For each test problem, the data points fall

Cosmos $^{\mathrm{TM}}$ is a registered trademark of Structural Research and Analysis Corporation (Los Angeles, CA). 
almost exactly on a straight line. For different problems, the slopes of these lines may be different, depending on the values of the second derivatives of the theoretical solution.

For these test problems, results for the minimum singular value criterion (Fig. 4) are very similar to the results based on the maximum eigenvalue criterion (see [4]). However, for elements with higher aspect ratios the minimum singular value condition is generally more accurate [7].

\section{CONCLUSIONS}

1. The minimum singular value of the 'edge shape matrix' of a tetrahedral element provides a link between several algebraic and geometric accuracy criteria, as specified below.

2. The minimum singular value of the edge shape matrix characterizes the "flatness" of a tetrahedron.

3. At the same time, the square of this minimum singular value is reciprocal to the maximum eigenvalue of the scaled edge stiffness matrix in the subspace corresponding to conservative fields. The latter eigenvalue governs the accuracy of FE approximation on an edge element.

4. The theoretical results provide a precise a priori accuracy criterion that can be applied locally (element-wise) or globally (to the whole mesh). The minimum singular value of the edge shape matrix $E$ can be replaced with the square root of the trace of $\left(E E^{T}\right)^{-1}$ for simplicity. The computational overhead in the overall $\mathrm{FE}$ procedure is negligible.

5. The minimum singular value criterion characterizes the Whitney edge element approximation of conservative fields or, equivalently, the first order node element approximation of the potential.

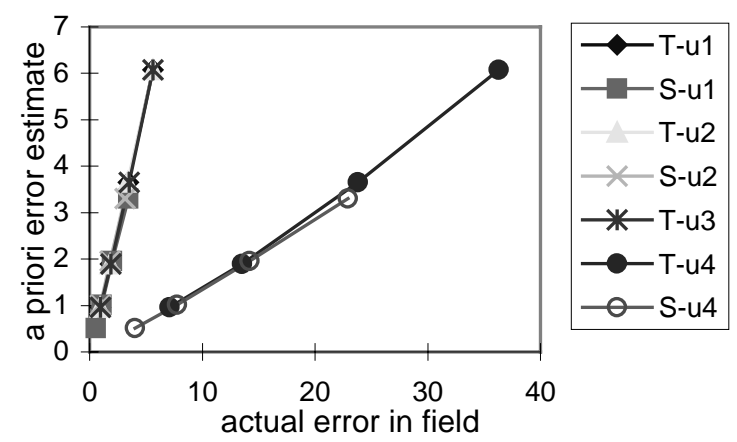

Fig. 4. Actual vs. estimated error for several test problems. Two mesh families are labeled with $T, S$; four test potentials are labeled u1-u4. See Appendix II.

\section{ACKNOWLEDGMENT}

I am very grateful to Dr. Alain Bossavit for inspiring this study and to Dr. Pierre Asselin for useful discussions. Dr. Asselin has obtained some related results by extending the methodology of Synge [2]. I would like to thank Alexander
Plaks for reading the manuscript and suggesting some revisions, and the anonymous reviewers for helpful comments.

\section{Appendix I. E-Matrix Can Have a Greater $\sigma_{\min }$ Than $G$-Matrix}

Consider a family of tetrahedra with the nodes represented in the Cartesian system as $(0,0,0) ;(1, \varepsilon, \varepsilon) ;(0,0, \varepsilon) ;$ and $(1,0, \varepsilon)$, where $\varepsilon>0$ is a parameter.

Then a direct calculation ${ }^{4}$ shows that in the limit $\varepsilon \rightarrow 0$ the singular value spectrum of $G$ is $\{\sqrt{2}, \sqrt{2}, 0\}$; i.e. $\sigma_{\min }(G) \rightarrow 0$. At the same time, in the same limit $\varepsilon \rightarrow 0$ the singular value spectrum of $E$ tends to $\{2,1,1\}$ (keep in mind that the columns of $E$ are unit vectors). That is, $\sigma_{\min }(E) \rightarrow 1$, which, due to (11), guarantees convergence of $\mathrm{FE}$ interpolation. A criterion based on $\sigma_{\min }(G)$ would incorrectly qualify elements with a small $\varepsilon$ as unacceptable.

\section{Appendix II. Brief Description of Test Problems}

Three meshes, $T_{2-4}$, are obtained by successive global refinements of the initial coarse mesh $T_{1}$ with 27 nodes and 44 elements $\left(T_{4}\right.$ then has 4,577 nodes and 22,528 elements). Another four meshes $S_{1-4}$, obtained in a similar way, have from 125 nodes and 375 elements to 35,181 nodes and 192,000 elements. Four test potentials u1-u4 satisfying the Laplace equation (with the appropriate inhomogeneous Dirichlet boundary conditions) are specified below:

$$
\begin{array}{lc}
\mathrm{u} 1=\sin x \sin y \sinh (\sqrt{2} z) & \mathrm{u} 3=\sin z \sin x \sinh (\sqrt{2} y) \\
\mathrm{u} 2=\sin y \sin z \sinh (\sqrt{2} x) & \mathrm{u} 4=\sin 1.5 x \sin 1.5 y \sinh (1.5 \sqrt{2} z)
\end{array}
$$

A test problem is defined by the mesh $\left(T_{1-4}\right.$ or $\left.S_{1-4}\right)$ and by the test solution $(\mathrm{u} 1-\mathrm{u} 4)$.

\section{REFERENCES}

[1] M. Zlámal, "On the finite element method," Numer. Math., vol. 12, pp.394-409, 1968.

[2] J. L Synge, The Hypercircle in Mathematical Physics; a Method for the Approximate Solution of Boundary Value Problems, Cambridge: University Press, 1957, pp.209-213.

[3] I. Babuška, A.K. Aziz, "On the angle condition in the finite element method," SIAM J. Numer. Anal., vol. 13, No. 2, pp.214-226, 1976.

[4] I. Tsukerman, "A general accuracy criterion for finite element approximation," this journal.

[5] G. H. Golub, C. F. Van Loan, Matrix Computations, Baltimore: Johns Hopkins University Press, 1996.

[6] A. Bossavit, "Whitney forms: a class of finite elements for threedimensional computations in electromagnetism," IEE Proc., vol. 135A, No. 8, pp.493-500, 1988.

[7] I. Tsukerman, A. Plaks, "Comparison of accuracy criteria for approximation of conservative fields on tetrahedra," this journal.

[8] I. Tsukerman, A. Plaks, "Refinement strategies and approximation errors for tetrahedral elements," submitted to IEEE Trans. Magn. (Proceeedings of CEFC'98, Tucson, June 1998).

[9] A. Bossavit, Computational Electromagnetism, Academic Press, 1997.

\footnotetext{
${ }^{4}$ MACsYma symbolic algebra package was used to facilitate the analysis. MACSYMA ${ }^{\circledR}$ is a registered trademark of Macsyma Inc.
} 